\title{
Safety of neuroangiography and embolization in children: complication analysis of 697 consecutive procedures in 394 patients
}

\author{
Ning Lin, MD, ${ }^{1,2}$ Edward R. Smith, MD, ${ }^{2}$ R. Michael Scott, MD, ${ }^{2}$ and Darren B. Orbach, MD, $P h D^{3}$ \\ 1Department of Neurosurgery, Weill Cornell Medical Center, New York, New York; and 2Department of Neurosurgery \\ and ${ }^{3}$ Division of Interventional and Neurointerventional Radiology, Boston Children's Hospital and Harvard Medical School, \\ Boston, Massachusetts
}

OBJECT The safe treatment of children using catheter-based angiography and embolization poses unique challenges because of the technical factors regarding the size and fragility of access and target vessels, as well as unique pediatric cerebrovascular pathologies. The complication rates for neurointerventional procedures in children have not been established.

METHODS The records of a consecutive cohort of pediatric patients who underwent neuroangiography and/or embolization between 2007 and 2013 were reviewed retrospectively to identify both intraprocedural and postprocedural complications. Demographic and clinical risk factors were analyzed with a multivariate logistic regression model.

RESULTS The 697 consecutive procedures consisted of 429 diagnostic angiograms and 268 embolizations (mean age of patients 11.1 years; range 4 days to 18 years; 217 females). There were 130 intracranial, 122 extracranial, and 16 spinal embolizations. Pathologies included 28 intracranial arteriovenous malformations (AVMs), 12 spinal AVMs, 19 aneurysms, 29 vein of Galen malformations, 29 dural arteriovenous fistulas, 96 extracranial AVMs, 39 tumors, 3 strokes, and 13 others. Overall, 2 intraprocedural and 1 postprocedural complication $(0.7 \%)$ occurred in the diagnostic group, all of which were nonneurological events. In the embolization group, 7 intraprocedural and 11 postprocedural complications $(6.7 \%)$ were observed. Of these complications, 15 were nonneurological events (5.6\%), 1 was a short-term neurological event (0.4\%), and 2 were long-term neurological events $(0.7 \%)$.

CONCLUSIONS Neither the technical challenges posed by children's access and target vessels nor the unique neurovascular pathologies seen in children need result in an elevated morbidity rate related to neuroangiography and embolization. At a dedicated high-volume center, the complication rates may be lower than those for comparable procedures performed in adults.

http://thejns.org/doi/abs/10.3171/2015.2.PEDS14431

KEY WORDS cerebral angiography; neurointervention; complication analysis; pediatric vascular disorders

$C_{\text {of }}$ ATHETER-BASED cerebral angiography and embolization are important tools for the management is still considered the gold standard for the diagnosis of intracranial vascular pathologies, and these conditions are increasingly being treated through endovascular approaches. Although safety profiles for diagnostic and interventional neuroendovascular procedures in adults have been described, $2,4,5,11,12,14,16,23,24,30,32-34,36,38$ the complication rates for children during and after such procedures have not been well established. Children have unique anatomy and physiology, which renders cerebral angiography and intervention more challenging than analogous procedures in adults. Small access-vessel size constrains the choice of guide catheters and may lead to increased risk of vascular injury, which can result in short-term and long-term morbidity, such as leg-length discrepancy. These procedures have contrast and radiation dose limitations according to patient age and weight. Intracranial target vessels are also smaller in caliber than those in adults and are therefore more vulnerable to thrombosis from partial obstruction,

ABBREVIATIONS AVF = arteriovenous fistula; $\mathrm{AVM}=$ arteriovenous malformation; $\mathrm{DSA}=$ digital subtraction angiography; VOGM = vein of Galen malformation. SUBMITTED September 7, 2014. ACCEPTED February 20, 2015.

INCLUDE WHEN CITING Published online June 26, 2015; DOI: 10.3171/2015.2.PEDS14431.

DISCLOSURE The authors report no conflict of interest concerning the materials or methods used in this study or the findings specified in this paper. 
and they are more fragile, especially early in life. Recent reports have described the risks of endovascular embolization in children with specific individual diagnoses, ${ }^{1,6,10,31}$ but an overall evaluation of the safety profile of all neurointerventional procedures in children has not been performed. In the present study, we reviewed our experience with diagnostic cerebral angiography and neurointerventions in children to assess whether the aforementioned challenges translate into an elevated morbidity rate in pediatric patients.

\section{Methods}

\section{Patient Selection}

This study included a consecutive cohort of pediatric patients at a specialized children's referral center who underwent cerebral angiography and/or neurointerventions between September 2006 and October 2013. Cases that involved non-catheter-based procedures, such as sclerotherapy, were not included in the cohort. In accordance with an institutional review board-approved protocol, a patient database was created, and data were collected prospectively and reviewed retrospectively to obtain demographic and clinical information such as age, sex, vascular pathology, symptoms, and results of radiographic studies, including CT, MRI, MR angiography, and DSA. Both intraprocedural and postprocedural complications were identified, and each such event was categorized as a nonneurological, transient neurological, or long-term neurological complication.

Each patient who underwent embolization was routinely seen for follow-up 1-2 weeks after the procedure and, depending on the nature of the vascular pathology, for long-term follow-up at intervals from 3 months to 1 year. Any report by a patient or his or her parents of a neurological change or any demonstration of a change from baseline on neurological examination at follow-up triggered a formal consultation by our neurology service. Data from the clinical follow-ups, the durations of which ranged from a minimum of 6 months up to a maximum of 92 months, were available for all the patients during the preparation of this article. The radiation dosage and projected stochastic effects of radiation exposure for each patient were also recorded as part of the complication analysis for some of this cohort; these results were reported separately. ${ }^{22}$

\section{Neuroangiography and Embolization Protocol}

All interventional procedures and the overwhelming majority of diagnostic procedures were performed with the patient under general anesthesia. To reduce accessrelated femoral complications, a micropuncture technique and ultrasound guidance were routinely used. For infants who underwent re-treatment, the puncture sites were usually switched to avoid accessing the same femoral artery. Groin sheath and access catheters were always flushed continuously with heparinized saline (the flow rate was minimized to avoid fluid overload), and systemic heparin (20-50 U/kg) was given during the neurointerventions (barring acute hemorrhagic presentation). Nonionic lowosmolality contrast material was used, and the dosage was limited to $7 \mathrm{ml} / \mathrm{kg}$. Standard fluoroscopy and angiographic runs were performed with $50 \%$ contrast. The intraproce- dural fluid status was monitored by the anesthesiology team, and children were normally placed on regular maintenance intravenous fluids at a dose based on body weight. Arterial closure was achieved by manual compression, and a deep extubation technique was used to minimize coughing and limb movement during the 4 hours of postprocedural recumbency spent in the postanesthesia care unit. Moreover, multiple mechanical and operator-dependent variables were optimized (e.g., tight collimation, pulsed fluoroscopy, aggressive filtration, automated dose control) to reduce radiation exposure. These protocols were described in previous reports. ${ }^{7,22}$

\section{Complication Analysis}

For the purposes of this paper, we considered a complication to be a clinical event, and angiographic events with no clinical sequelae, such as an intraprocedural vasospasm that responded quickly to an administered calcium channel blocker, were not included in the complication tally. Differences in demographic and clinical characteristics were examined by using chi-square and 2-tailed t-tests for binary and continuous variables, respectively. The association between demographic and clinical risk factors and the periprocedural complication rate was evaluated with chi-square and univariate regression analyses. Multivariate logistic regression was used to calculate the odds ratios (ORs) and 95\% confidence intervals (95\% CIs) for the likelihood of complications after adjusting for age, sex, pathology, type of intervention, and re-treatment status. By "neurological complication," we refer to an adverse event that involved the CNS; thus, for example, a patch of skin with decreased sensation after embolization of a facial AVM was considered a nonneurological complication. The types of intervention were categorized as intracranial embolization, extracranial embolization, or spinal embolization, based on the site of primary pathology. Re-treatment status indicated whether a procedure was the first intervention for a particular patient or a repeated endeavor. Statistical significance was defined as a Type I error $(<0.05)$. All statistical analyses were performed using SAS version 9.2 (SAS Institute, Inc.) and Excel 2007 (Microsoft Corp.).

\section{Results}

Between September 2006 and October 2013, 697 consecutive neuroangiographic procedures were performed by one operator on 394 pediatric patients at our institution, and data were collected in our database; 429 of them were diagnostic angiograms, and 268 were neurointerventions. Demographic information is summarized in Table 1. The mean age of patients at time of the procedure was 11.1 years (range 4 days to 18 years), and 341 procedures were performed on female patients. Although more than half of the procedures involved children more than 10 years of age, the cohort also included 53 patients younger than 1 year, 13 of whom were less than 3 months old at the time of the procedure.

A variety of vascular and nonvascular pathologies were treated, and the angiographic diagnoses for the neurointerventional procedures are listed in Table 2 . There were 130 interventions for intracranial lesions, including 29 vein of 
Galen malformations (VOGMs), 28 arteriovenous malformations (AVMs), 29 arteriovenous fistulas (AVFs), and 19 aneurysms. Fifteen procedures were performed to deliver intraarterial chemotherapy for retinoblastoma. Extracranial vascular malformations were treated with embolization (99 cases), and the majority of the 22 extracranial tumors that were embolized were juvenile nasopharyngeal angiofibromas. The spinal vascular lesions consisted of 12 spinal AVFs or AVMs.

Overall, periprocedural complications occurred in 21 patients $(3.0 \%)$ in the entire cohort: $3(0.7 \%)$ in the diagnostic group and $18(6.7 \%)$ in the intervention group (Table $3)$. The majority of them were nonneurological events, such as groin site bleeding necessitating longer manual pressure (4 cases), hair loss likely related to positioning (3 cases), and brief intraembolization bradycardia from trigeminocardiac reflex (3 cases, with the first reported in a separate publication) ${ }^{26}$ One 14-month-old boy had a retained stretched and sheared microcatheter after Onyx embolization of infantile myofibromatosis in his neck, and his case was reported in a separate publication. ${ }^{25} \mathrm{~A}$ mild contrast reaction occurred in 2 children, and no patients developed contrast medium-related nephropathy. One patient, a 1-year-old girl with a VOGM who underwent transarterial embolization, experienced a postoperative transient neurological deficit, waking up with intermittent right lateral gaze palsy. This condition resolved completely without intervention within several hours after the procedure, and the patient was at her neurological baseline during the follow-up visits. There was 1 case of decreased femoral pulse at the access site that lasted for several weeks in a 3-month-old patient who underwent tumor embolization, but it resolved to baseline by 6 weeks (ultrasound revealed vessel patency). No long-term lower-extremity complications, such as iliofemoral injury or leg-length discrepancy, as reported in a separate publication, ${ }^{7}$ occurred in our cohort.

There were 2 long-term neurological complications (0.7\%); a 16-year-old girl developed intracranial hemorrhage after technically uncomplicated preoperative embolization of a brain AVM and required emergent craniotomy, and a 17-year-old girl with dwarfism related to Seckel syndrome and moyamoya disease suffered a thromboembolic event during aneurysm coiling. The former patient had near-complete recovery from the adverse event and had minimal long-term morbidity (she initially had hemiparesis and ultimately recovered her normal strength but had residual mild hyperreflexia). The latter patient had permanent neurological morbidity (hemiparesis).

Univariate analyses were conducted to identify risk factors associated with any complications and factors associated specifically with neurological complications; the results are shown in Table 4. In terms of complications overall, females were more likely than males to have periprocedural complications ( $8.9 \%$ vs $4.2 \%$, respectively; $\mathrm{p}=$ 0.02 ), and first-time interventional procedures were associated with a higher rate of complication than re-treatments (9.6\% vs $3.0 \%$, respectively; $p=0.01$ ). Those with a diagnosis of tumor or AVF carried a higher risk of complication. Patients who underwent intracranial embolization had a statistically significantly higher rate of complication
TABLE 1. Demographic characteristics on admission

\begin{tabular}{lccc}
\hline & \multicolumn{2}{c}{ Procedure } & \\
\cline { 2 - 3 } \multicolumn{1}{c}{ Variable } & $\begin{array}{c}\text { Diagnostic } \\
\text { Angiography } \\
(\mathrm{n}=429)\end{array}$ & $\begin{array}{c}\text { Neurointervention } \\
(\mathrm{n}=268)\end{array}$ & $\begin{array}{c}\text { All Patients } \\
(\mathrm{n}=697)\end{array}$ \\
\hline Mean age (range) & $\begin{array}{c}\text { 10.8 yrs (14 } \\
\text { days to 18 }\end{array}$ & $\begin{array}{c}11.7 \text { yrs }(4 \text { days to } \\
18 \text { yrs })\end{array}$ & $\begin{array}{c}11.1 \text { yrs }(4 \\
\text { days to } \\
\end{array}$ \\
yrs $)$ & & 18 yrs $)$ \\
\hline Age distribution (no.) & & & \\
$<3$ mos & 6 & 7 & 13 \\
3 mos to 1 yr & 17 & 23 & 40 \\
1 to 10 yrs & 179 & 85 & 264 \\
$>10$ yrs & 227 & 153 & 380 \\
\hline Sex (no.) & & & \\
$\quad$ Female & 217 & 124 & 341 \\
Male & 212 & 144 & 356 \\
\hline
\end{tabular}

than those who underwent extracranial or spinal embolization ( $10.0 \%$ vs $3.3 \%$ and $0.0 \%$, respectively; $p=0.04$ ). A trend toward young (3 months to 1 year old, $8.7 \%$ ) and very young $(<3$ months old, $14.3 \%)$ patients having more nonneurological complications than older children was observed, although statistical significance was not reached.

In terms of neurological complications specifically, all 3 were in female patients $(p=0.007)$. No evidence suggested that young age was a factor in neurological complications; 2 of the 3 events occurred in children $>10$ years of age. Intracranial embolization may carry a higher risk of neurological complications than extracranial embolization and spinal embolization, although this difference was not statistically significant $(2.3 \%$ vs $0.0 \%$ and $0.0 \%$, respectively; $p=0.24$ ).

In a multivariable logistic regression model, age, sex, pathology, re-treatment status, and type of intervention were evaluated as independent variables. Intracranial embolization (OR 8.5; 95\% CI 1.86-39.2) and diagnosis of

TABLE 2. Angiographic diagnoses for neurointerventional procedures

\begin{tabular}{lc}
\hline Angiographic Diagnosis & No. of Procedures $(\mathrm{n}=268)$ \\
\hline Intracranial & 130 \\
VOGM & 29 \\
AVM & 28 \\
AVF & 29 \\
Aneurysm & 19 \\
Stroke & 3 \\
Tumor (embolization) & 1 \\
Tumor (chemotherapy) & 15 \\
Other & 6 \\
\hline Extracranial & 122 \\
AVM/AVF & 96 \\
Tumor & 22 \\
Other & 4 \\
\hline Spinal & 16 \\
AVM/AVF & 12 \\
Tumor & 1 \\
Aneurysmal bone cyst & 3 \\
\hline
\end{tabular}


TABLE 3. Periprocedural complications in diagnostic and interventional cases*

\begin{tabular}{|c|c|c|c|}
\hline \multirow[b]{2}{*}{ Complication } & \multicolumn{2}{|l|}{ Procedure } & \multirow[b]{2}{*}{$\begin{array}{l}\text { All Patients } \\
(n=697)\end{array}$} \\
\hline & Diagnostic Angiography $(n=429)$ & $\begin{array}{l}\text { Neurointervention } \\
\quad(n=268)\end{array}$ & \\
\hline All & $3(0.7)$ & $18(6.7)$ & $21(3.0)$ \\
\hline Nonneurological & 3 & 15 & 18 \\
\hline Contrast allergy & 2 & 0 & 2 \\
\hline Heparin hypersensitivity & 0 & 1 & 1 \\
\hline Hair loss & 1 & 2 & 3 \\
\hline Bradycardia & 0 & 3 & 3 \\
\hline Groin site bleeding & 0 & 4 & 4 \\
\hline Decreased pulse at groin site & 0 & 1 & 1 \\
\hline Coil/Onyx migration & 0 & 2 & 2 \\
\hline Retained catheter & 0 & 1 & 1 \\
\hline Numb patch after facial embolization & 0 & 1 & 1 \\
\hline Transient neurological & 0 & 1 & 1 \\
\hline Cranial nerve palsy & 0 & 1 & 1 \\
\hline Nontransient neurological & 0 & 2 & 2 \\
\hline Ischemic stroke & 0 & 1 & 1 \\
\hline Hemorrhage & 0 & 1 & 1 \\
\hline
\end{tabular}

${ }^{*}$ Values represent the number (\%) of procedures.

tumor (OR 6.8; 95\% CI 1.32-35.6) were associated with any periprocedural complication, whereas young age, female sex, and re-treatments were not significant risk factors (Table 5).

\section{Discussion}

With growing recognition of pediatric cerebrovascular disorders and advances in the endovascular armamentar-

\section{TABLE 4. Any complications and neurological complications after a neurointerventional procedure}

\begin{tabular}{|c|c|c|c|c|}
\hline $\begin{array}{l}\text { All Patients w/ Intervention }(n=268) \\
\text { (no. of patients) }\end{array}$ & Any Complication $(n=17)^{*}$ & p Value & Neurological Complication $(n=3)^{*}$ & $p$ Value \\
\hline \multicolumn{5}{|l|}{ Age } \\
\hline$<3 \operatorname{mos}(7)$ & $1(14.3)$ & \multirow[t]{4}{*}{0.71} & $0(0.0)$ & \multirow[t]{4}{*}{0.86} \\
\hline 3 mos to 1 yr (23) & $2(8.7)$ & & $0(0.0)$ & \\
\hline 1 to 10 yrs $(85)$ & $6(7.1)$ & & $1(1.2)$ & \\
\hline$>10$ yrs (153) & $8(5.2)$ & & $2(2.0)$ & \\
\hline \multicolumn{5}{|l|}{ Sex } \\
\hline Female (124) & $11(8.9)$ & \multirow[t]{2}{*}{0.02} & $3(2.4)$ & \multirow[t]{2}{*}{0.007} \\
\hline Male (144) & $6(4.2)$ & & $0(0.0)$ & \\
\hline \multicolumn{5}{|l|}{ Re-treatment status } \\
\hline First intervention (135) & $13(9.6)$ & \multirow[t]{2}{*}{0.01} & $2(1.5)$ & \multirow[t]{2}{*}{0.32} \\
\hline Re-treatment (133) & $4(3.0)$ & & $1(0.8)$ & \\
\hline \multicolumn{5}{|l|}{ Diagnosis } \\
\hline Aneurysm (19) & $1(5.3)$ & \multirow[t]{7}{*}{0.34} & $1(5.3)$ & \multirow[t]{7}{*}{0.68} \\
\hline $\operatorname{AVF}(35)$ & $4(11.4)$ & & $0(0.0)$ & \\
\hline Tumor (39) & $5(12.8)$ & & $0(0.0)$ & \\
\hline $\operatorname{VOGM}(29)$ & $2(6.9)$ & & $1(3.4)$ & \\
\hline Aneurysmal bone cyst (3) & $0(0.0)$ & & $0(0.0)$ & \\
\hline Other (13) & $0(0.0)$ & & $0(0.0)$ & \\
\hline AVM (130) & $5(3.8)$ & & $1(1.5)$ & \\
\hline \multicolumn{5}{|l|}{ Type of intervention } \\
\hline Intracranial embolization (130) & $13(10.0)$ & \multirow[t]{3}{*}{0.04} & $3(2.3)$ & \multirow[t]{3}{*}{0.24} \\
\hline Spinal embolization (16) & $0(0.0)$ & & $0(0.0)$ & \\
\hline Extracranial embolization (122) & $4(3.3)$ & & $0(0.0)$ & \\
\hline
\end{tabular}

* Values represent the number (\%) of patients (number of patients is specified parenthetically in the far-left column). 
TABLE 5. Multivariate logistic regression for factors associated with any complications or neurological complications after a neurointerventional procedure

\begin{tabular}{|c|c|c|}
\hline \multirow[b]{2}{*}{ Factor } & \multicolumn{2}{|c|}{$\mathrm{OR}(95 \% \mathrm{Cl})$} \\
\hline & Any Complication & $\begin{array}{l}\text { Neurological } \\
\text { Complication }\end{array}$ \\
\hline Age, $<1 \mathrm{yr}$ & $1.3(0.3-6.0)$ & $0.0(0.0-999)$ \\
\hline Sex, female & $2.2(0.6-7.7)$ & $999(0.0-999)$ \\
\hline $\begin{array}{l}\text { Re-treatment status, re- } \\
\text { treated }\end{array}$ & $0.3(0.1-1.1)$ & $0.2(0.01-4.0)$ \\
\hline Diagnosis, tumor & $6.8(1.32-35.6)$ & $0.002(0.0-999)$ \\
\hline $\begin{array}{l}\text { Type of intervention, in- } \\
\text { tracranial embolization }\end{array}$ & $8.5(1.86-39.2)$ & $2.4(0.1-45.5)$ \\
\hline
\end{tabular}

ium, catheter-based cerebral angiography and neurointerventional procedures have been performed with increasing frequency in children. ${ }^{3}$ However, nearly all clinical trials related to neuroendovascular treatments are conducted in adult populations, ${ }^{17-20,35}$ which results in a paucity of information regarding the neuroendovascular safety profile in children. Not only are pediatric patients anatomically and physiologically different from adults, they also harbor unique cerebrovascular pathologies rarely seen in adults (e.g., VOGMs), and either of these factors can theoretically affect procedure-related morbidity. Therefore, it is impossible to extrapolate the safety profile of cerebral angiography and neurointerventions from adults to children.

Three previous studies reported on the safety of diagnostic cerebral angiography at high-volume pediatric centers. Burger et al. ${ }^{3}$ retrospectively reviewed 241 consecutive diagnostic angiograms in 205 children and reported no intraprocedural complications, 2 minor postprocedural complications (groin site bleeding), and 1 postprocedural hemorrhage $(0.4 \%)$. Wolfe et al. ${ }^{37}$ reviewed a series of 46 cerebral angiograms on 42 patients and reported no periprocedural complications. Hoffman et al. ${ }^{9}$ reviewed a series of 309 cerebral angiograms performed in 87 children $<36$ months of age; 292 of the angiograms were performed for intraarterial chemotherapy. They reported a nonneurological complication rate of $2.9 \%$ and no neurological complications. Our results in the diagnostic group of our series (429 angiograms) corroborate and extend these findings, with 2 minor intraprocedural events (contrast allergy) and 1 postprocedural event (hair loss), for a total complication rate of $0.7 \%$, and they all resolved without long-term consequences. We saw no neurological complications, and there were no deaths. These results collectively suggest that diagnostic cerebral angiography can be conducted safely at high-volume centers with an extremely low risk of vascular or systemic complications in children, even those of a very young age.

In addition, a number of recent studies focused on the perioperative risks of specific endovascular interventions in smaller cohorts of children and reported relatively high morbidity. Saraf et al. ${ }^{27}$ treated 23 cerebral aneurysms in children with endovascular embolization and reported 3 postprocedural ischemic strokes and 1 hemorrhage (17\%). Ashour et al. ${ }^{1}$ performed 105 Onyx embolization proce- dures on 69 pediatric patients with a vascular lesion or tumor and reported 25 total complications, including 7 cases of ischemic stroke, 3 hemorrhages, and 3 vessel perforations. In contrast, the rate of long-term neurological complications in our interventional group was extremely low $(0.7 \%)$. The 2 cases of major complications in our cohort exemplified different aspects of the technical challenges related to neurointerventions in children. In 1 case, the patient was a 17-year-old girl with a history of dwarfism related to Seckel syndrome (she weighed $14 \mathrm{~kg}$ ) and of moyamoya syndrome. The patient underwent endovascular coiling for an unruptured growing posterior communicating artery aneurysm and suffered intraprocedural parent vessel thrombosis. Although this complication can certainly occur in adults, the unique anatomy in this patient posed severe technical challenges that increased both the risk of thrombosis and the difficulties around attempted rescue maneuvers. In contradistinction, the other patient was a 16-year-old girl with a right frontal AVM who underwent technically uneventful elective preoperative embolization in preparation for resection the following day; she suffered intracerebral hemorrhage overnight and required emergent craniotomy and resection. This type of complication is in no way unique to children, with the adult literature reporting the risk of postembolization hemorrhage after AVM embolization to be $4 \%-12 \% .8,13,21,23,31$ Putative causes of postembolization hemorrhage in adults, such as a high volume of embolic agent administered, ${ }^{23}$ are likely equally applicable to children.

In our experience, several factors contributed to the low complication rate after the neurointerventions we report here. First, our approach is intrinsically interdisciplinary; literally every case is reviewed by our team, with both open surgical and endovascular options weighed; with the full panoply of options available at our institution, we are not biased toward one approach over the other. Second, information available from cross-sectional imaging is maximally used to avoid unnecessary vessel access, angiographic runs, and, in some circumstances, diagnostic angiography altogether. Rather than adopting adult goals, we design our treatment goals to be age appropriate and tailored specifically for children (e.g., staging embolization of a complex high-flow AVM or AVF to avoid massive hemodynamic shifts). Careful padding is essential to avoid positioning-related iatrogenic adverse events. Heavy emphasis is placed on frequent, open, and thorough communication among the team of dedicated pediatric care providers during preprocedural planning, intraprocedurally, and postprocedurally.

In terms of technical approach, fastidious adherence to our protocol for managing groin access as a function of patient age and weight protected the patients in our cohort against permanent iliofemoral injury or leg-length discrepancy, as we previously reported. . Similarly, we adhered rigorously to a contrast dose limit of $7 \mathrm{ml} / \mathrm{kg}$ to avoid iodinated contrast nephropathy and neurotoxicity. There has been no systematic study of appropriate dose limits for iodinated contrast for neuroangiography in children; the widely accepted norms for dose limits currently used throughout radiology are based on an expert committee opinion published in $1977,{ }^{29}$ with no explicit scientific ba- 
sis elucidated. A recent review of a cohort of 2321 children who underwent interventional cardiac procedures, ${ }^{28}$ with administered doses of contrast up to a maximum of 16.8 $\mathrm{ml} / \mathrm{kg}$, reported only 2 cases of morbidity attributable to contrast; neither of these patients were in the quartile with the highest contrast dose (>6 ml/kg, 580 cases). Using this dose limit of $7 \mathrm{ml} / \mathrm{kg}$, we have encountered no cases of contrast-related nephropathy or neuropathy. Finally, there may be no substitute for a practice environment with a team that is devoted nearly exclusively to treating children and therefore performs pediatric neuroangiography and embolization procedures on a regular basis.

Considering all types of periprocedural complications, we found that females carried significantly higher risk than males and that intracranial embolization presented higher risk than extracranial or spinal embolization. Considering neurological complications in particular, female sex was again a statistically significant factor. It is interesting to note that a perhaps related observation was reported from the study of a large cohort of patients who underwent coronary angiography; women were found to have a significantly higher risk of periprocedural complication..$^{15}$ Children who underwent repeat interventions had a lower risk of overall complications than those who were undergoing their first interventional procedure. Age, surprisingly, was not a statistically significant factor for periprocedural or neurological complications according to the univariate and multivariate analyses. A diagnosis of tumor was associated with a higher rate of overall complications (5 of $39[12.8 \%]$ ), although these events were mostly minor and were all nonneurological; the 5 complications that occurred in patients with a tumor consisted of 2 episodes of bradycardia, 2 episodes of groin site bleeding, and 1 retained catheter (Table 4).

The major weakness of this study, as has been the case for all previous studies of neurointerventional morbidity in adults and of morbidity with diagnostic angiography in children, is its retrospective nature. Moreover, followup evaluation was performed by the treating physician; thus, the examinations were not routinely performed by a third party. However, any report by the parents or child of a deviation from neurological baseline or the elicitation of any deviation from neurological baseline on examination triggered a formal consultation by the neurology service. Finally, in young children treated while still achieving neurodevelopmental milestones (e.g., an infant who is undergoing embolization of a VOGM), it is intrinsically difficult or impossible to disentangle late-appearing milestones or disabilities (e.g., slight language delay as a toddler or learning disabilities in grade school) as effects of the underlying pathology versus possible treatment morbidity.

\section{Conclusions}

Our results suggest that in high-volume centers that routinely treat pediatric patients, neurointerventional procedures can be performed with a safety profile in children as good as or better than that in adults. Neither the unique cerebrovascular conditions treated nor the anatomical and physiological challenges in the pediatric population need translate into increased procedural morbidity.

\section{References}

1. Ashour R, Aziz-Sultan MA, Soltanolkotabi M, Schoeneman SE, Alden TD, Hurley MC, et al: Safety and efficacy of Onyx embolization for pediatric cranial and spinal vascular lesions and tumors. Neurosurgery 71:773-784, 2012

2. Brinjikji W, McDonald JS, Kallmes DF, Cloft HJ: Rescue treatment of thromboembolic complications during endovascular treatment of cerebral aneurysms. Stroke 44:1343-1347, 2013

3. Burger IM, Murphy KJ, Jordan LC, Tamargo RJ, Gailloud P: Safety of cerebral digital subtraction angiography in children: complication rate analysis in 241 consecutive diagnostic angiograms. Stroke 37:2535-2539, 2006

4. Chen J, Gailloud P: Safety of spinal angiography: complication rate analysis in 302 diagnostic angiograms. Neurology 77:1235-1240, 2011

5. Dawkins AA, Evans AL, Wattam J, Romanowski CA, Connolly DJ, Hodgson TJ, et al: Complications of cerebral angiography: a prospective analysis of 2,924 consecutive procedures. Neuroradiology 49:753-759, 2007

6. Gobin YP, Dunkel IJ, Marr BP, Brodie SE, Abramson DH: Intra-arterial chemotherapy for the management of retinoblastoma: four-year experience. Arch Ophthalmol 129:732737, 2011

7. Gross BA, Orbach DB: Addressing challenges in $4 \mathrm{~F}$ and $5 \mathrm{~F}$ arterial access for neurointerventional procedures in infants and young children. J Neurointerv Surg 6:308-313, 2014

8. Haw CS, terBrugge K, Willinsky R, Tomlinson G: Complications of embolization of arteriovenous malformations of the brain. J Neurosurg 104:226-232, 2006

9. Hoffman CE, Santillan A, Rotman L, Gobin YP, Souweidane MM: Complications of cerebral angiography in children younger than 3 years of age. J Neurosurg Pediatr 13:414419, 2014

10. Jabbour P, Chalouhi N, Tjoumakaris S, Gonzalez LF, Dumont AS, Chitale R, et al: Pearls and pitfalls of intraarterial chemotherapy for retinoblastoma. J Neurosurg Pediatr 10:175-181, 2012

11. Kaufmann TJ, Huston J III, Mandrekar JN, Schleck CD, Thielen KR, Kallmes DF: Complications of diagnostic cerebral angiography: evaluation of 19,826 consecutive patients. Radiology 243:812-819, 2007

12. Kondo R, Matsumoto Y, Endo H, Miyachi S, Ezura M, Sakai $\mathrm{N}$ : Endovascular embolization of cerebral arteriovenous malformations: results of the Japanese Registry of Neuroendovascular Therapy (JR-NET) 1 and 2. Neurol Med Chir (Tokyo) 54:54-62, 2014

13. Ledezma CJ, Hoh BL, Carter BS, Pryor JC, Putman CM, Ogilvy CS: Complications of cerebral arteriovenous malformation embolization: multivariate analysis of predictive factors. Neurosurgery 58:602-611, 2006

14. Leonardi M, Cenni P, Simonetti L, Raffi L, Battaglia S: Retrospective study of complications arising during cerebral and spinal diagnostic angiography from 1998 to 2003. Interv Neuroradiol 11:213-221, 2005

15. Lichtman JH, Wang Y, Jones SB, Leifheit-Limson EC, Shaw LJ, Vaccarino V, et al: Age and sex differences in inhospital complication rates and mortality after percutaneous coronary intervention procedures: evidence from the NCDR. Am Heart J 167:376-383, 2014

16. Lv X, Wu Z, Jiang C, Li Y, Yang X, Zhang Y, et al: Complication risk of endovascular embolization for cerebral arteriovenous malformation. Eur J Radiol 80:776-779, 2011

17. McDougall CG, Spetzler RF, Zabramski JM, Partovi S, Hills NK, Nakaji P, et al: The Barrow Ruptured Aneurysm Trial. J Neurosurg 116:135-144, 2012

18. Mohr JP, Moskowitz AJ, Stapf C, Hartmann A, Lord K, Marshall SM, et al: The ARUBA trial: current status, future hopes. Stroke 41:e537-e540, 2010

19. Molyneux A, Kerr R, Stratton I, Sandercock P, Clarke M, 
Shrimpton J, et al: International Subarachnoid Aneurysm Trial (ISAT) of neurosurgical clipping versus endovascular coiling in 2143 patients with ruptured intracranial aneurysms: a randomised trial. Lancet 360:1267-1274, 2002

20. Molyneux AJ, Kerr RS, Yu LM, Clarke M, Sneade M, Yarnold JA, et al: International subarachnoid aneurysm trial (ISAT) of neurosurgical clipping versus endovascular coiling in 2143 patients with ruptured intracranial aneurysms: a randomised comparison of effects on survival, dependency, seizures, rebleeding, subgroups, and aneurysm occlusion. Lancet 366:809-817, 2005

21. Morgan MK, Winder M, Little NS, Finfer S, Ritson E: Delayed hemorrhage following resection of an arteriovenous malformation in the brain. J Neurosurg 99:967-971, 2003

22. Orbach DB, Stamoulis C, Strauss KJ, Manchester J, Smith ER, Scott RM, et al: Neurointerventions in children: radiation exposure and its import. AJNR Am J Neuroradiol 35:650-656, 2013

23. Ovalle F, Shay SD, Mericle RA: Delayed intracerebral hemorrhage after uneventful embolization of brain arteriovenous malformations is related to volume of embolic agent administered: multivariate analysis of 13 predictive factors. Neurosurgery 70 (2 Suppl Operative):313-320, 2012

24. Pierot L, Cognard C, Herbreteau D, Fransen H, van Rooij WJ, Boccardi E, et al: Endovascular treatment of brain arteriovenous malformations using a liquid embolic agent: results of a prospective, multicentre study (BRAVO). Eur Radiol 23:2838-2845, 2013

25. Puri AS, Rahbar R, Dearden J, Graham RJ, Lillehei C, Orbach DB: Stretched and sheared microcatheter retained after Onyx embolization of infantile myofibromatosis. Interv Neuroradiol 17:261-266, 2011

26. Puri AS, Thiex R, Zarzour H, Rahbar R, Orbach DB: Trigeminocardiac reflex in a child during pre-Onyx DMSO injection for juvenile nasopharyngeal angiofibroma embolization. A case report. Interv Neuroradiol 17:13-16, 2011

27. Saraf R, Shrivastava M, Siddhartha W, Limaye U: Intracranial pediatric aneurysms: endovascular treatment and its outcome. J Neurosurg Pediatr 10:230-240, 2012

28. Senthilnathan S, Gauvreau K, Marshall AC, Lock JE, Bergersen L: Contrast administration in pediatric cardiac catheterization: dose and adverse events. Catheter Cardiovasc Interv 73:814-820, 2009

29. Shehadi WH: Contrast media in diagnostic radiology: recommendations for labels, package inserts, and dosage determination. AJR Am J Roentgenol 129:167-170, 1977

30. Shigematsu T, Fujinaka T, Yoshimine T, Imamura H, Ishii A, Sakai C, et al: Endovascular therapy for asymptomatic unruptured intracranial aneurysms: JR-NET and JR-NET2 findings. Stroke 44:2735-2742, 2013

31. Soltanolkotabi M, Schoeneman SE, Alden TD, Hurley MC, Ansari SA, DiPatri AJ Jr, et al: Onyx embolization of intracranial arteriovenous malformations in pediatric patients. J Neurosurg Pediatr 11:431-437, 2013

32. Stapf C: The rationale behind "A Randomized Trial of Unruptured Brain AVMs" (ARUBA). Acta Neurochir Suppl 107:83-85, 2010

33. Thiex R, Norbash AM, Frerichs KU: The safety of dedicatedteam catheter-based diagnostic cerebral angiography in the era of advanced noninvasive imaging. AJNR Am J Neuroradiol 31:230-234, 2010

34. Waldau B, Turk AS III, Yashar P, Khaldi A, Turner RD IV, Chaudry MI, et al: Perioperative safety of Hydrosoft coils. J Neurointerv Surg 4:375-378, 2012

35. Wiebers DO, Whisnant JP, Huston J III, Meissner I, Brown RD Jr, Piepgras DG, et al: Unruptured intracranial aneurysms: natural history, clinical outcome, and risks of surgical and endovascular treatment. Lancet 362:103-110, 2003

36. Willinsky RA, Taylor SM, TerBrugge K, Farb RI, Tomlinson G, Montanera W: Neurologic complications of cerebral angiography: prospective analysis of 2,899 procedures and review of the literature. Radiology 227:522-528, 2003

37. Wolfe TJ, Hussain SI, Lynch JR, Fitzsimmons BF, Zaidat OO: Pediatric cerebral angiography: analysis of utilization and findings. Pediatr Neurol 40:98-101, 2009

38. Yamada NK, Cross DT III, Pilgram TK, Moran CJ, Derdeyn CP, Dacey RG Jr: Effect of antiplatelet therapy on thromboembolic complications of elective coil embolization of cerebral aneurysms. AJNR Am J Neuroradiol 28:1778-1782, 2007

\section{Author Contributions}

Conception and design: Orbach, Lin. Acquisition of data: Lin. Analysis and interpretation of data: Lin. Drafting the article: Lin. Critically revising the article: all authors. Reviewed submitted version of manuscript: all authors. Approved the final version of the manuscript on behalf of all authors: Orbach. Statistical analysis: Lin. Administrative/technical/material support: Orbach. Study supervision: Orbach.

\section{Correspondence}

Darren B. Orbach, Neurointerventional Radiology, Boston Children's Hospital, 300 Longwood Ave., CB 1113, Boston, MA 02115. email: darren.orbach@childrens.harvard.edu. 